American Journal of Pharmaceutical Education 2021; 85 (3) Article 8287.

\title{
COMMENTARY
}

\section{Addressing Burnout in Pharmacy Residency Programs}

\author{
Jordan M. Potter, PharmD, Megan L. Cadiz, PharmD \\ Beaumont Hospital, Royal Oak, Michigan \\ Submitted July 12, 2020; accepted November 23, 2020; published March 2021.
}

\begin{abstract}
Addressing health care provider burnout has become a top priority for many health care systems. Unfortunately, a paucity of literature is available on preventing and managing burnout in pharmacy residents despite an increase in articles on the prevalence of pharmacy burnout. This limits the ability of pharmacy managers to understand and address burnout in this high-risk population. Until further investigation into the most beneficial, evidence-based strategies for managing burnout in this population is conducted, this commentary offers strategies to address and mitigate burnout in pharmacy residency training programs based on the available interdisciplinary literature.

The authors have first-hand experience with burnout and aim to begin a paradigm shift to emphasize well-being and reshape the culture of postgraduate training. Dr. Potter has dedicated time to developing programming and founded an interprofessional well-being organization called Mindfulness in Newly Developing Students of Healthcare (MINDS) for health care students and providing recommendations for pharmacy residency programs. As a program director, Dr. Cadiz focuses on the development of realistic and effective strategies to eliminate burnout and foster well-being in her pharmacy residency training program.
\end{abstract}

Keywords: burnout, pharmacy resident, well-being, postgraduate training

\section{INTRODUCTION}

Health care provider burnout has become a major topic of interest across all disciplines because of the longlasting consequences of burnout on clinicians' careers, health care systems, and patient care. ${ }^{1-4}$ The uptick in publications related to clinician burnout has improved the working knowledge of health administrators and program directors on the prevalence of, risk factors for, and potential interventions to address this problem. Historically, burnout was thought to only impact health care practitioners late in their professional careers. However, more recent evidence suggests that burnout affects health care providers in all stages of their career, as early as their didactic education. ${ }^{3}$ Within pharmacy education, special consideration must be given to pharmacists completing residency programs as residents are likely to experience the multiple professional and personal stressors associated with postgraduate training and exhibit many factors associated with burnout.

Unfortunately, a paucity of literature on burnout in pharmacy residents is available. This limits the Academy's ability to understand and address burnout in this

Corresponding Author: Jordan M. Potter, Beaumont Hospital - Royal Oak, 3601 W 13 Mile Rd., Royal Oak, MI 48073. Tel: 270-217-4160. Email: Jordan.Potter@ optioncare.com at-risk population. The American Association of Colleges of Pharmacy (AACP) issued the following statement on well-being: "AACP believes that all administrators, faculty, staff, preceptors, student pharmacists and alumni should contribute to a culture of wellness and resilience in pharmacy education." "As an organization that commits itself to advancing and transforming pharmacy education across the career continuum from student to new practitioner, it is imperative for the AACP to shine light on this important issue.

We hope this commentary will serve as a call to action for the Academy to improve our working knowledge of pharmacy resident burnout and evidence-based strategies to improve resident well-being. The utility of this manuscript is multifactorial. For residency programs, it may serve as a framework within which to establish wellbeing initiatives aimed at improving the pharmacy practice and learning environment. Academic faculty and preceptors may garner new strategies from this article to use to promote well-being and reduce burnout in their learners. Finally, individuals considering or currently completing residency training may gain insight into potential strategies they may implement or attributes they may look for in a residency program that suggest the administrators are aware of the dangers of burnout and have a burnout prevention program in place. 


\section{American Journal of Pharmaceutical Education 2021; 85 (3) Article 8287.}

\section{DISCUSSION}

\section{Understanding Burnout}

Burnout is a job-related syndrome that a person develops as the result of prolonged response to chronic interpersonal stressors. ${ }^{6}$ Over time, the person's inability to recover from work-related stress manifests as one or more of three burnout subdomains: emotional exhaustion, depersonalization, and reduced personal accomplishment. ${ }^{1,7}$ Health care provider burnout has been associated with diminished well-being, decreased job satisfaction and performance, substance abuse or dependence, depression, and suicidality. ${ }^{1-3,6,8,9}$ In addition to affecting clinicians personally, burnout has a significant impact on the health care systems where they work and the patient care they provide. Medical errors, mortality, and infections have been associated with provider burnout as well, demonstrating its negative impact on patient care. ${ }^{1,2,8}$

The Collaborative on Clinician Well-Being and Resilience reports that $45 \%$ to $60 \%$ of medical students and residents suffer from substantial symptoms related to burnout. ${ }^{1}$ As a profession, pharmacists may be predisposed to burnout as a result of regulatory compliance, excessive documentation, and incongruities between training and daily tasks. ${ }^{8,10}$ The prevalence of burnout in pharmacy residents has not been reported directly; however, based on the findings from several studies, rates of pharmacist burnout are likely from $46 \%$ to $62 \%$. $^{9-11}$

Health system administrators and pharmacy residency directors should understand the factors that influence the development and/or severity of burnout, identify at-risk individuals, and employ appropriate training and well-being strategies. Individual factors associated with increased risk of burnout include: younger age, female gender, having young children or no children, being unmarried, perceived stress, hours worked, days on call, and moral distress. ${ }^{1,4,7,9-13}$ Professional stressors nearly double the risk of burnout and include having inadequate administrative and teaching time, uncertainty about health care reform, difficult colleagues, too many nonclinical duties, and underappreciated contributions. ${ }^{9}$ Lack of autonomy and control, heavy workload, no or minimal margin for error, interruption-driven environments, workplace disorganization, and ambiguity in the work itself are all early predictors of burnout related to the practice and learning environment. ${ }^{1,4,7,9-13}$

These risk factors provide a glimpse into why many clinicians are predisposed to burnout. However, the specific risk factors for pharmacy residents have not been defined. Residency training in itself is an incredible stressor and has been associated with burnout, increased perceived stress and incidence of depression, and decreased quality of life. ${ }^{12-14}$ Moreover, residents are typically young, in their first pharmacist role, spend long hours on site, and often participate in on-call programs, where they may be faced with traumatic and stressful medical emergencies. The new practitioners' pursuit of accelerated training through residency and progressive development requires substantial time and effort, potentially reducing dedicated time for well-being activities known to reduce burnout. ${ }^{4,13}$ Additionally, the increase in responsibilities, expectations, and pressure to excel all place residents under greater professional, situational, and personal stress. ${ }^{4}$

\section{Addressing Pharmacy Resident Burnout}

Resident well-being is the cornerstone to combatting burnout in pharmacy residency training programs. Wellbeing has been described as the presence of positive and absence of negative emotions, satisfaction with life, fulfillment, and positive functioning. ${ }^{1}$ By definition, wellbeing is a psychological state subject to change from moment to moment. Well-being can be addressed by implementing new programs, improving the practice and/ or learning environment, and supporting individual wellbeing efforts. The Guidance Document for the ASHP Accreditation Standard For Postgraduate Year One (PGY1) Pharmacy Residency Programs provided by the American Society of Health-System Pharmacists (ASHP) were updated in 2019 to include statements about burnout and well-being. ${ }^{15}$ Implementation of these standards, however, is left up to each residency program. As such, many programs may struggle to integrate well-being initiatives into their curricula despite having a strong desire to do so. Even with the availability of well-being initiatives, residents may be averse to using such programming because of pressures to achieve, meet expectations, and/or obtain recommendations for jobs or second-year training programs. Others may fear that admitting they have a problem and/or seeking help will have implications on their licensure and jeopardize their career. Routine discussion of burnout with residents in a group setting may help destigmatize burnout and empower trainees to explore and adopt strategies to mitigate burnout and improve well-being.

Currently, many well-being strategies emphasize improving individual coping skills. Individual-based approaches are undeniably important, but evidence outlines the importance of creating positive practice and/or learning environments, providing support, and developing interventions at the system and organizational levels. ${ }^{16,17}$ Unfortunately, the implementation of organization-wide well-being initiatives has not been wellestablished and reports of implementation are limited to 


\section{American Journal of Pharmaceutical Education 2021; 85 (3) Article 8287.}

Table 1. Evidence Summary of Several Proposed Strategies to Improve the Well-Being of Pharmacy Residents

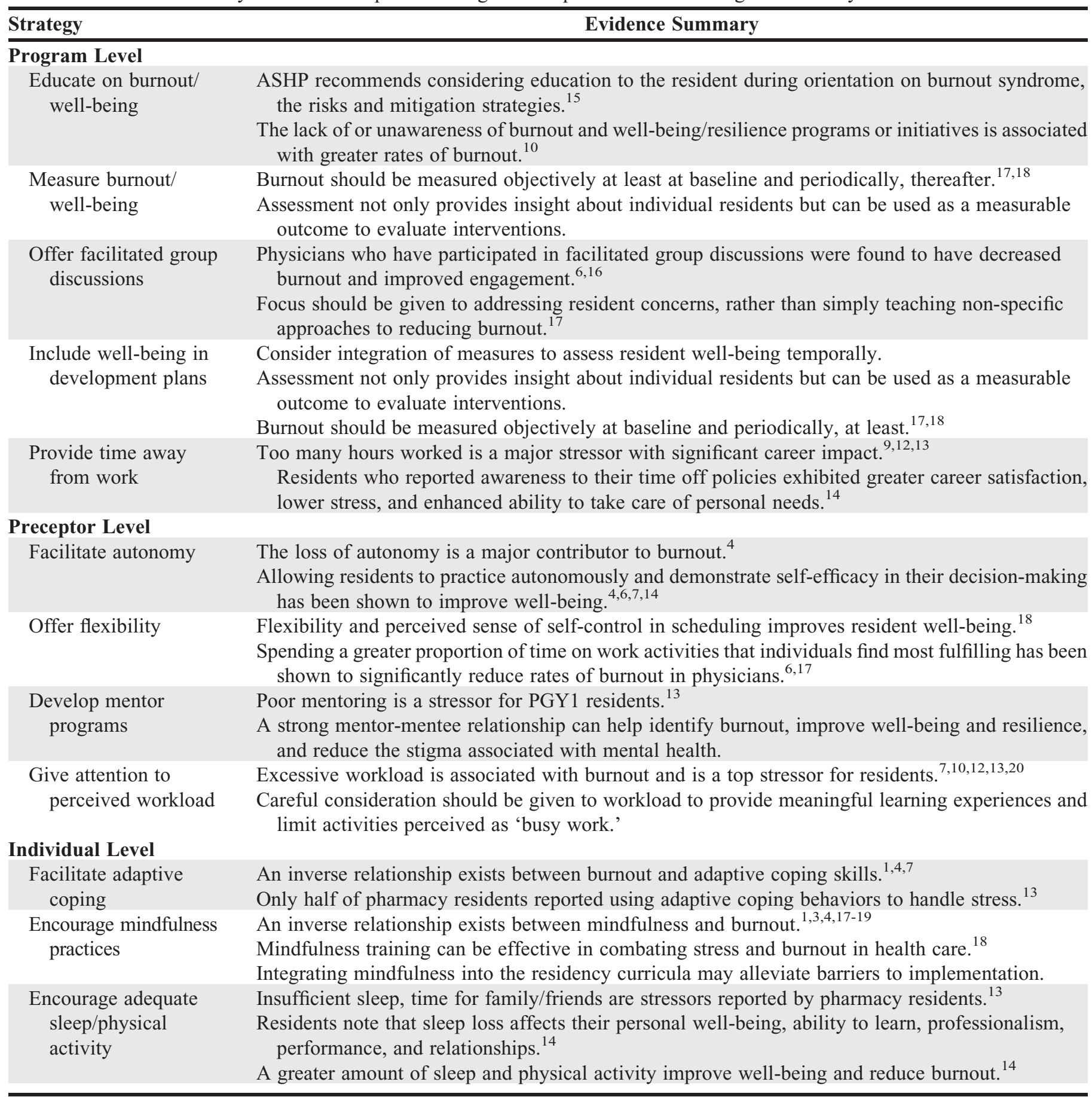

several case studies. ${ }^{10}$ The National Academy of Medicine has developed a conceptual model to highlight the importance of clinician well-being and further illustrate the necessity of adaptation in the practice and/or learning environment. ${ }^{7}$ While the most effective strategies have not been determined, residency programs can address burnout by supporting initiatives at the program, preceptor, and individual levels. Below we propose several strategies based on the available interdisciplinary literature. A brief summary of the supporting evidence is provided in Table 1. No one strategy is necessarily better than another; thus, programs administrators should consider developing a combination of strategies tailored to the specific needs of their program and residents. 


\section{American Journal of Pharmaceutical Education 2021; 85 (3) Article 8287.}

\section{Strategies}

Receiving continual program support is paramount to residents understanding burnout, improving their wellbeing, and reshaping the culture of postgraduate training. During the orientation period, first-year residents should be educated about the prevalence and warning signs of burnout, as well as made aware of mitigation strategies and resources for well-being and resilience. ${ }^{15}$ Programs should also consider holding a well-being retreat or conference as a part of orientation and establish seminar-type lectures related to well-being. ${ }^{1,6,16,18,19}$ Regular follow-up sessions should be offered throughout the program to remind residents of the resources available to them.

Another strategy to consider is hosting facilitated group discussions. These sessions offer clinicians the chance to share and process experiences that may be stressful and/or traumatic., ${ }^{6}$ Simultaneously, group discussions provide a safe environment and may initiate a paradigm shift in the stigma associated with burnout. Such sessions may also provide the opportunity for health system administrators and residency directors to recognize the signs and symptoms of burnout early and intervene as appropriate.

Integration of well-being measures into the resident development plan provides an opportunity for program administrators to emphasize the importance of residents' well-being and ensure regular assessment of residents for signs of burnout. Further, programs may want to consider integrating well-being and/or burnout assessment into learning experience evaluations. This encourages residents to reflect on their self-care and well-being practices and provides program administrators with insight into the resident's well-being.

Burnout is characterized by the inability of a person to recover from the stress of work-related events. It can be inferred that removing oneself from the work environment enables recovery from burnout. Time away from work also provides residents with time to practice selfcare and physical well-being. Residents should be made aware of the program's time-off policy during orientation and encouraged to use the provided time to prevent burnout and improve their well-being.

Completion of postgraduate training through residency requires oversight and a coordinated program to meet the rigorous ASHP standards. As a result, most programs are highly structured and offer residents limited flexibility and personal choice. However, autonomy, flexibility, and a sense of control are protective factors against burnout. ${ }^{11}$ For pharmacy residents, autonomous decisions may take the form of topic selection for a research project or continuing education lecture, availability for and number of elective rotations, and selection of their mentor or advisor. Potential interventions to broaden flexibility include allowing residents to alter their schedule as their career goals change and developing projects and presentations based on residents' individual areas of interest.

In addition to embracing autonomy and flexibility, preceptors can significantly impact resident well-being and resilience through mentorship and advising, serving as a well-being role model, giving attention to the resident's perceived workload, and offering recognition for resident success. ${ }^{6,11,13,16}$ Specifically, mentoring is a crucial component of professional development and residents can especially benefit in the transition from student to new practitioner. ${ }^{11}$ Potential roles include aid with relocation, navigation of the licensure process, rotation selection, and professional development. Other roles may be residentspecific and dependent on the resident's career goals, practice interests, identified strengths, and areas for improvement. In addition to advising, positive modeling behaviors can be impactful. ${ }^{17}$ These behaviors include employing personal well-being and resilience techniques, maintaining positive work-life integration, and demonstrating interests outside of work. Preceptors should be encouraged to share their own experiences with burnout and mitigation strategies if they feel comfortable doing so.

The resident's perception of workload has major implications on their personal and professional lives. ${ }^{11,13,20}$ Those involved in the training of residents should involve the resident in establishing expectations and activities to align the learning experience with the needs, career goals, and expectations of the learner. As a program, all longitudinal requirements should be frequently reviewed to ensure each is enriching, supportive of the program objectives, and meaningful for the resident and program.

Current evidence outlines the importance of strategies, but it would be imprudent to neglect the importance of individual well-being efforts. ${ }^{1,16}$ Effectively managing their personal stress is vital to residents' well-being. However, not all coping strategies are healthy or advisable. Despite temporarily providing perceived stress relief, maladaptive coping strategies can negatively impact burnout and well-being. ${ }^{20}$ Examples of maladaptive coping include disengagement, denial, self-blame, alcohol use, and substance abuse/dependence. Conversely, adaptive coping mechanisms such as planning, positive reframing, active coping, acceptance, and use of support help alleviate stress and encourage well-being. ${ }^{20}$ Top coping strategies reported by pharmacy residents include spending time with family and friends, staying optimistic, and engaging in other activities they find meaningful. ${ }^{13}$

Mindfulness has been shown to improve career satisfaction and reduce stress, burnout, and anxiety and 


\section{American Journal of Pharmaceutical Education 2021; 85 (3) Article 8287.}

depression. ${ }^{1,3,4,17-19}$ Informal activities that usually take less than 10 minutes have great utility in health care as these exercises can be conducted "on the fly." Typically, these involve pausing and grounding oneself in the present moment.

Finally, getting adequate sleep and dedication to sleep hygiene are imperative for proper functioning and physiologic restoration. Pharmacy residents are expected to perform at high levels during their training, yet many pharmacy residents report not getting enough sleep. ${ }^{13}$ Obtaining sufficient, quality sleep should be an individual priority and one that is supported by the residency curricula, including having an enforceable duty hours policy in place. ${ }^{6}$ In addition to proper sleep, engaging in physical activity improves quality of life and decreases burnout in postgraduate training. ${ }^{6}$ Scheduling the activity or coordinating group activities helps maintain accountability, but the most beneficial duration or type of activity to reduce burnout has not been identified.

\section{CONCLUSION}

Addressing burnout in pharmacy resident training programs will require a paradigm shift to emphasize wellbeing and reshape the culture of postgraduate training. Pharmacy residents may be especially prone to the detrimental effects of burnout because of the presence of risk factors and increased stress and responsibility associated with postgraduate training. Unfortunately, a paucity of literature on this subject exists and further investigation is necessary to identify the most effective strategies to promote pharmacy resident well-being and reduce burnout. The development and maintenance of well-being initiatives at the program, preceptor, and individual levels is essential to support progressive advancement of new practitioners and facilitate the delivery of high-quality patient care. Future research and collaboration will be required to develop realistic and effective strategies to eliminate burnout and foster well-being in pharmacy residency training programs.

\section{REFERENCES}

1. National Academies of Sciences Engineering and Medicine. 2019. Taking action against clinician burnout: a systems approach to professional well-Being. Washington, DC: The National Academies Press. https://doi.org/10.17226/25521.

2. Dyrbye LN, Shanafelt TD, Sinsky C. Burnout among health care professionals: a call to explore and address this underrecognized threat to safe, high-quality care. NAM Perspectives. 2017;7(7).

3. West CP, Dyrbye LN, Shanafelt TD. Physician burnout: contributors, consequences and solutions. J Intern Med. 2018;283(6): 516-529.
4. Chaukos D, Chad-Friedman E, Mehta DH, et al. Risk and resilience factors associated with resident burnout. Acad Psychiatry. 2017;41(2):189-194.

5. American Association of Colleges of Pharmacy AACP Statement on Commitment to Clinician Well-being and Resilience. https:// www.aacp.org/article/commitment-clinician-well-being-andresilience. Accessed March 3, 2021.

6. Lam N-C, Black E. Implementing wellness curriculum in residency. In: Contemporary Topics in Graduate Medical Education. 2018.

7. Brigham T, Barden A, Dopp A. A journey to construct an allencompassing conceptual model of factors affecting clinician wellbeing and resilience. NAM Perspectives. 2018;8(1).

8. Bridgeman PJ, Bridgeman MB, Barone J. Burnout syndrome among healthcare professionals. Am J Health Syst Pharm. 2018; 75(3): 147-152.

9. Jones GM, Roe NA, Louden L, et al. Factors associated with burnout among US hospital clinical pharmacy practitioners: results of a nationwide pilot survey. Hosp Pharm. 2017;52(11): $742-751$

10. Durham ME, Bush PW, Ball AM. Evidence of burnout in healthsystem pharmacists. Am J Health Syst Pharm. 2018;

75(23_Supplement_4):S93-S100.

11. McQuade B, Reed B, DiDomenico R, et al. Feeling the burn? A systematic review of burnout in pharmacists. $\mathrm{J} \mathrm{Am} \mathrm{Coll} \mathrm{Clin} \mathrm{Pharm.}$ 2020;3(3):663-675.

12. Le HM, Young SD. Evaluation of stress experienced by pharmacy residents. Am J Health Syst Pharm. 2017;74(8):599-604. 13. Zinurova E, DeHart R. Perceived stress, stressors, and coping mechanisms among PGY1 pharmacy residents. Am J Pharm Educ. 2018;82(7):6574.

14. Raj KS. Well-Being in residency: a systematic review. J Grad Med Ed. 2016;8(5):674-684.

15. American Societey of Health-System Pharmacists. ASHP Accreditation Standard for Postgraduate Year One (PGY1) Pharmacy Residency Programs. https://www.ashp.org/-/media/assets/ professional-development/residencies/docs/guidance-documentPGY1-standards.ashx. Accessed March 3, 2021.

16. West CP, Dyrbye LN, Erwin PJ, et al. Interventions to prevent and reduce physician burnout: a systematic review and meta-analysis. Lancet. 2016;388(10057):2272-2281.

17. Sinsky C, Daugherty Biddison A, Mallick A, et al. Organizational Evidence-Based and Promising Practices for Improving Clinician Well-Being. Discussion Paper, National Academy of Medicine, Washington, DC. NAM Perspectives. 2020. https://nam.edu/organizational-evidence-based-and-promisingpractices-for-improving-clinician-well-being/. Accessed March 3, 2021.

18. Linzer M, Levine R, Meltzer D, et al. 10 bold steps to prevent burnout in general internal medicine. J Gen Intern Med. 2014;29(1): $18-20$.

19. Panagioti M, Panagopoulou E, Bower P, et al. Controlled interventions to reduce burnout in physicians: a systematic review and meta-analysis. JAMA Intern Med. 2017;177(2):195-205. 20. Sum MY, Chew QH, Sim K. Perceptions of the learning environment on the relationship between stress and burnout for residents in an ACGME-I Accredited National Psychiatry Residency Program. J Grad Med Ed. 2019;11(4 Suppl):85-90. 